\title{
MÉTODOS EXTRATORES E FITODISPONIBILIDADE DE ZINCO PARA MILHO EM ARGISSOLO VERMELHO-AMARELO EUTRÓFICO
}

\author{
CAPACITY OF THE EXTRATION METHODS AND BIOAVAILABILITY \\ OF ZINC FOR CULTURE OF THE MAIZE CULTIVATED IN EUTROPHIC \\ YELLOW RED ARGISOL
}

\author{
Affonso Celso GONÇALVES JR*(1,3) \\ Ângela Lemos PRESTES $(2,3,4)$ \\ Ossival Lolato RIBEIRO $(2,3)$ \\ Alexandre Leseur dos SANTOS ${ }^{(2,3)}$
}

\begin{abstract}
RESUMO
O aumento da produtividade de grãos, com redução dos custos, depende atualmente do uso de micronutrientes como complementação da adubação convencional. Neste estudo foram testados dois extratores químicos (Mehlich-1 e DTPA), na extração de Zn disponível em Argissolo Vermelho-Amarelo eutrófico, além de verificar os teores deste metal no tecido foliar de milho cultivado neste solo, com aplicação de diferentes doses de Zn sob diferentes tratamentos com NPK. O experimento foi conduzido na cidade de Palotina - PR (Setembro/2002 - Janeiro/2003), em delineamento experimental de blocos casualizados, com quatro repetições. Os tratamentos foram dispostos em esquema fatorial constituído de três formas de adubação NPK: sem adubação (testemunha); uma vez a recomendação de adubação para a cultura do milho e o dobro da dose recomendada de adubação. As fontes de $\mathrm{P}, \mathrm{K}$ e Zn na base foram fosfato bicálcico, nitrato de potássio e sulfato de zinco, respectivamente. As doses de Zn utilizadas foram: 0,0;5,0 e 10,0 $\mathrm{mg} \mathrm{dm}^{-3}$. Para análise química, coletou-se 10 folhas por parcela num total de 36 amostras, e também amostras de solo na linha e entrelinha, perfazendo num total 72 amostras. A determinação de N, P e K foi realizada segundo metodologia do IAPAR, e a determinação de Zn foi realizada por digestão nitro-peróxido e técnicas de espectrometria de absorção atômica de chama. Os extratores mais utilizados para determinação dos teores de Zn no solo são o Mehlich-1 e DTPA, sendo que ambos demonstraram uma boa correlação entre os teores presentes no solo e sua fitodisponibilidade no tecido foliar do milho.
\end{abstract}

Palavras-chave: Extratores; Zinco; Milho.

\begin{abstract}
Increasing grain yielder, with reduction of costs, depends currently on the use micronutrients as complementation of the conventional fertilization. In this study two chemical extractors were tested (Mehlich-1 and DTPA), in the extration of Zn available in eutrophic Yellow Red Argisol, besides verifying amount of this metal in the foliate fabric of maize cultivated in this soil, with application of different doses of Zn under different treatments with NPK. The experiment was lead in the city of Palotina - PR (September/2002 January/2003), in a randomized block delineation, with four replication. The treatments had been made in constituted factorial project of three forms of fertilization NPK: without fertilization (check); one time the recommendation of fertilization for the culture of the maize and the double of the recommended dose of fertilization. The sources of $\mathrm{P}, \mathrm{K}$ and $\mathrm{Zn}$ in the base had been phosphate bicalcium potassium nitrate and zinc sulphate, respectively. The used doses of $Z n$ were: 0,$0 ; 5,0$ and 10,0 $\mathrm{mg} \mathrm{dm}^{-3}$. For chemical analysis, it was collected 10 leaves for parcel in a total of 36 samples, and also soil samples in the line and space between lines, totalizing a total 72 samples. The determination of $\mathrm{N}, \mathrm{P}$ and $\mathrm{K}$ were accomplished to methodology of the IAPAR, and the determination of $\mathrm{Zn}$ was accomplished by digestion nitro-peroxide and techniques of spectrometry of atomic absorption of flame. The extractors used for determination of amount of $\mathrm{Zn}$ in the soil are the Mehlich-1 and DTPA, being that both had demonstrated a good correlation among the present amount in the soil and its bioavailability in the foliate fabric of the maize.

Key-words: Extractors; Zinc; Maize.
\end{abstract}

\footnotetext{
${ }^{1}$ Químico Dr., Professor do Centro de Ciências Agrárias - UNIOESTE (*e-mail: affonso133@hotmail.com)

${ }^{2}$ Acadêmico do Centro de Ciências Agrárias da UNIOESTE.

${ }^{3}$ Grupo de Estudos em Solos e Meio Ambiente (GESOMA). Endereço: Rua Pernambuco, 1777, CEP 85.960-000. Marechal Cândido Rondon - PR.

${ }^{4}$ Trabalho de Conclusão de Curso/ PIBIC.
} 


\section{INTRODUÇÃO}

O aumento da produtividade dos grãos devese atualmente também à prática do uso de micronutrientes, que tem se intensificado nos últimos. As pesquisas sobre disponibilidade de nutrientes, particularmente micronutrientes em solos tem apresentado resultados contraditórios. Isto se deve, em grande parte, às numerosas inter-relações entre as características do solo e a porção do nutriente tida com disponível para as culturas $(1,2)$. Tais resultados indicam a necessidade de maior refinamento das interpretações que melhor expressem a disponibilidade de micronutrientes e a resposta das plantas (13).

O Zn, em especial na cultura do milho, está relacionado com enzimas responsáveis pelo crescimento da planta, pois o milho armazena todos os seus compostos no caule e folha desde a germinação até a fase reprodutiva, que é quando os compostos serão utilizados no enchimento de grão (6). O teor de $\mathrm{Zn}$ nas plantas varia bastante, dependendo das espécies e de fatores do solo. Normalmente, os teores encontram-se na faixa de 20 a $50 \mathrm{mg} \mathrm{kg}^{-1}$, sendo freqüentes sintomas de deficiência quando as plantas apresentam teores menores do que $20 \mathrm{mg} \mathrm{kg}^{-1}$. É clássica na literatura, a deficiência de $\mathrm{Zn}$ induzida por altos níveis de $\mathrm{P}$ no substrato, principalmente em solos ricos em óxido de $\mathrm{Fe}$ e $\mathrm{Al}$, devido à adsorção do $\mathrm{Zn}(3,4)$. Entretanto, a adubação com micronutrientes não pode ser feita de maneira indiscriminada, deve-se avaliar a sua disponibilidade para evitar gastos desnecessários e efeitos fitotóxicos pelo excesso de aplicação (10).

Para proceder-se à análise dos nutrientes no solo para fins de adubação, utilizam-se reagentes químicos geralmente soluções diluídas, que simulam a ação das raízes das plantas, ou seja, devem extrair do solo apenas as formas químicas dos nutrientes que as raízes extraem. A força extratora dessas soluções varia conforme a substância utilizada, sua concentração e a proporção solo/solução que se utiliza durante a extração (14).

Um bom extrator, conforme McLaughlin (12) deverá: (a) ser relativamente simples e de baixo custo; (b) ser calibrado sobre condições de campo em diferentes tipos de solos; (c) considerar o maior número de fatores do ambiente que reconhecidamente influenciam as concentrações dos metais nas culturas; e (d) ser preditivo - além de avaliar a disponibilidade atual dos metais, ele deverá prever a disponibilidade ao longo do desenvolvimento da cultura.

Diversos trabalhos, citados por Galrão (5), relatam a busca de extratores confiáveis para diversas culturas, defrontando-se com a influência do próprio extrator no $\mathrm{pH}$ do solo. Assim, extratores ácidos, como Mehlich-1, podem extrair mais $\mathrm{Zn}$ do solo, podendo extrair níveis superiores aos disponíveis para as plantas, já os extratores complexantes, como o DTPA, extraem menos $\mathrm{Zn}$ do solo, apontando para maior eficácia do primeiro em solos mais ácidos e do segundo para solos mais alcalinos.
De acordo com Abreu e Raij (1), que estudaram o efeito da reação do solo no $Z$ n extraído pelas soluções de DTPA e Mehlich-1, o extrator DTPA mostrou melhores resultados com valores altos e positivos de correlação entre os teores de Zn na planta e no solo que o extrator Mehlich-1, concluindo que a mudança na disponibilidade de Zn, decorrente da reação do solo, foi mais bem avaliada pela solução de DTPA.

De acordo com Korndörfer (9), que testou formas de adição de $\mathrm{Zn}$ a um formulado NPK e seu efeito sobre a produção de milho, os resultados mostraram que o $\mathrm{Zn}$ agregado ao adubo NPK não teve efeito sobre a produção de grãos de milho, nem sobre a produção de matéria seca, independentemente da dose ou da fonte empregada. Os teores de $\mathrm{Zn}$ na folha do milho aumentaram significativamente com as doses de $\mathrm{Zn}$ aplicadas.

Portanto, este trabalho foi realizado com o objetivo de testar dois extratores químicos, Mehlich-1 $\left(\mathrm{HCl} 0,05 \mathrm{~mol} \mathrm{~L}^{-1}+\mathrm{H}_{2} \mathrm{SO}_{4} 0,05 \mathrm{~mol} \mathrm{~L}^{-1}\right)$ e o agente complexante DTPA (Dietileno triamino penta-acético), na extração do Zn disponível em Argissolo VermelhoAmarelo eutrófico textura média, além de verificar os teores de $\mathrm{Zn}$ no tecido vegetal de milho cultivado neste solo, com diferentes doses de $\mathrm{Zn}$ sob diferentes tratamentos com NPK.

\section{METODOLOGIA}

O experimento foi conduzido na Cidade de Palotina - PR, em delineamento experimental de blocos casualizados, com quatro repetições em Argissolo Vermelho-Amarelo eutrófico textura média $(24,2 \%$ de argila, $64,0 \%$ de areia e $11,8 \%$ de silte).

O híbrido de milho empregado foi Agroeste, híbrido simples 1544 de ciclo precoce. Cada bloco apresentava $6 \mathrm{~m}$ de largura por $33 \mathrm{~m}$ de comprimento. Utilizou-se como parcela útil, as duas linhas centrais ( $3 \mathrm{~m}$ de cada linha), das quatro semeadas em espaçamento de $0,90 \mathrm{~m}$, descartando-se $1,5 \mathrm{~m}$ de bordadura das duas linhas centrais. A população de plantas foi de 6,5 sementes por metro linear e o experimento foi instalado em setembro de 2002.

Os tratamentos foram dispostos em esquema fatorial constituído de três formas de adubação NPK: sem adubação (testemunha); uma vez a recomendação de adubação para a cultura do milho através da análise de rotina $\left(30-100-50 \mathrm{~kg} \mathrm{ha}^{-1}\right) \mathrm{e}$ o dobro da dose recomendada de adubação (60 $\left.200-100 \mathrm{~kg} \mathrm{ha}^{-1}\right)$. As doses de $\mathrm{Zn}$ utilizadas foram: 0,$0 ; 5,0$ e $10,0 \mathrm{mg} \mathrm{kg}^{-1}$. Os tratamentos foram aplicados no momento da semeadura. As fontes de $\mathrm{N}, \mathrm{P}, \mathrm{K}$ e $\mathrm{Zn}$ na base foram uréia, fosfato bicálcico, nitrato de potássio e sulfato de $\mathrm{Zn}$.

A semeadura do milho foi em sistema convencional, com uma subsolagem e duas gradagens, além de capinas manuais para o controle de plantas daninhas. Com 45 dias após a emergência, foi aplicada em cobertura a adubação nitrogenada 
sendo utilizada como fonte a uréia $(45 \% \mathrm{~N})$, com dose igual para todas as parcelas, $90 \mathrm{~kg} \mathrm{ha}^{-1}$ de $\mathrm{N}$.

Realizou-se análise química completa do solo antes da semeadura, a qual apresentou os resultados contidos na Tabela 1, segundo metodologia utilizada pelo IAPAR (8).

TABELA 1 - Análise química do solo.

\begin{tabular}{|c|c|c|c|c|c|c|c|c|c|c|c|c|c|c|c|}
\hline$P$ & $\mathrm{MO}$ & $\mathrm{pH}$ & $\mathrm{H}+\mathrm{Al}$ & $\mathrm{Al}^{3+}$ & $\mathrm{K}^{+}$ & $\mathrm{Ca}^{2+}$ & $\mathrm{Mg}^{2+}$ & SB & CTC & $\mathrm{V}$ & $\mathrm{Al}$ & $\mathrm{Cu}$ & $\mathrm{Zn}$ & $\mathrm{Fe}$ & $\mathrm{Mn}$ \\
\hline $\begin{array}{l}\text { Mg.dm } \\
\text { Mehlich-1 }\end{array}$ & g.dm ${ }^{-3}$ & $\begin{array}{c}\mathrm{CaCl}_{2} \\
0,01 \mathrm{~mol} \mathrm{~L}^{-1}\end{array}$ & ----------. & 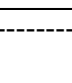 & $-----c$ & $\mathrm{nol}_{\mathrm{c}} \mathrm{dr}$ & -3- & - & ----- & $\%$ & & ------- & $--m g$ & $\mathrm{dm}^{-3}$ & - \\
\hline 2,41 & 15,41 & 5,01 & 6,21 & 0 & 0,19 & 5,29 & 2,51 & 7,9 & 14,2 & 56 & 0 & 2,0 & 2,7 & 19 & 61 \\
\hline
\end{tabular}

A coleta do material vegetal para análise química foi realizada no florescimento, coletando-se 10 folhas por parcela (folha oposta e abaixo da espiga principal), num total de 36 amostras, que foram secas, moídas e armazenadas. Juntamente com a coleta de tecido vegetal foram realizadas coletas de amostras de solo sendo três sub-amostras na linha de plantio e três sub-amostras na entrelinha. As amostras da linha foram misturadas formando uma amostra composta. O mesmo foi feito com as amostras da entrelinha, perfazendo num total 72 amostras. Estas foram secas (48 $\mathrm{h}$ a $\left.65^{\circ} \mathrm{C}\right)$ e peneiradas $(<0,2 \mathrm{~mm})$.

A extração de $Z n$ do solo foi realizada nas 72 amostras utilizando-se os extratores Mehlich-1 e DTPA. As determinações dos teores de N, P e K no tecido foliar foram realizadas segundo metodologia do IAPAR (7), e a determinação de Zn foliar foi realizada por digestão nitro-peróxida e técnica de espectrometria de absorção atômica modalidade chama.

\section{RESULTADOS E DISCUSSÃO}

Os dados obtidos foram submetidos à análise de variância e as médias comparadas pelo teste de Tukey a $5 \%$ de probabilidade.

$A$ análise de variância demonstrou efeito da interação entre as doses de $\mathrm{Zn}$ e adubação NPK (Tabela 2). Pelo desdobramento da interação, verificou-se que na maior dose de $\mathrm{Zn}\left(10 \mathrm{mg} \mathrm{dm}^{-3}\right.$ ) aplicada no solo, ocorreu maior disponibilização deste elemento no tecido foliar mas de acordo com o incremento da adubação NPK, pois a medida que aumentaram-se as doses de NPK diminuiram-se os teores de $\mathrm{Zn}$ na folha. Isso pode ser justificado pelo efeito da diluição do $\mathrm{Zn}$ em relação à adubação com NPK ou a efeitos antagônicos entre $\mathrm{P}$ e $\mathrm{Zn}$.

TABELA 2 - Teores de Zn no tecido foliar de acordo com o desdobramento da interação adubação NPK - Zn.

\begin{tabular}{|c|c|c|c|}
\hline $\begin{array}{c}\text { Zn aplicado } \\
\left(\mathrm{mg}^{\left.-\mathrm{dm}^{-3}\right)}\right.\end{array}$ & & $\begin{array}{c}\mathrm{N}-\mathrm{P}_{2} \mathrm{O}_{5}-\mathrm{K} \\
\left(\mathrm{kg} \cdot \mathrm{ha}^{-1}\right) \\
\end{array}$ & \\
\hline $\begin{array}{c}0 \\
5 \\
10\end{array}$ & $\begin{array}{c}0 \\
21,8 \text { b B } \\
33,5 \text { a A } \\
40,2 \text { a A }\end{array}$ & $\begin{array}{c}30-100-50 \\
24,0 \text { b AB } \\
25,0 \text { b B } \\
37,2 \text { a } A B\end{array}$ & $\begin{array}{c}60-200-100 \\
26,0 \text { a } A \\
28,7 \text { a } A B \\
30,0 \text { a B }\end{array}$ \\
\hline $\begin{array}{c}\text { DMS } \\
\text { CV (\%) }\end{array}$ & & $\begin{array}{c}4,6655 \\
15,59\end{array}$ & \\
\hline
\end{tabular}

É freqüente na literatura a deficiência de Zn causada por altos níveis de $P$ no substrato, principalmente em solos ricos em óxidos de $\mathrm{Fe}$ e Al, devido à adsorção do Zn. Malavolta (11) cita que a deficiência de $\mathrm{Zn}$ induzida pelo $\mathrm{P}$ é conhecida na literatura há muito tempo e a aplicação de adubos fosfatados freqüentemente intensifica uma deficiência marginal de Zn limitando a produção. Segundo o autor várias explicações são utilizadas para entender esse fenômeno: o P diminui a absorção do Zn por um processo de inibição; o excesso de $\mathrm{P}$ absorvido reduz a quantidade de $Z n$ que da raiz vai para a parte aérea, dependendo do $\mathrm{pH}$ e da concentração de fosfato no meio poderá haver precipitação do $\mathrm{Zn}$ pelo $\mathrm{P}$ na superfície das raízes; a adição do $P$ provoca maior produção de matéria seca e, portanto, diluição no teor de $\mathrm{Zn}$ no tecido que pode cair abaixo do nível adequado quando então a deficiência aparece e o crescimento fica limitado pela falta do micronutriente.

Os resultados de $\mathrm{Zn}$ obtidos no solo com os diferentes extratores (Mehlich-1 e DTPA) são apresentados nas tabelas 3 e 4 . Com relação ao $\mathrm{Zn}$ extraído por Mehlich-1 (Tabela 3), as doses de 5 e 10 $\mathrm{mg} \cdot \mathrm{dm}^{-3}$ de $\mathrm{Zn}$ foram as que apresentaram os valores mais elevados deste metal, diferenciando-se estatisticamente da testemunha. 
GONÇALVES JR, A.C. et al. Métodos extratores e fitodisponibilidade...

TABELA 3 - Teores médios de Zn extraídos do solo pelo extrator Mehlich-1.

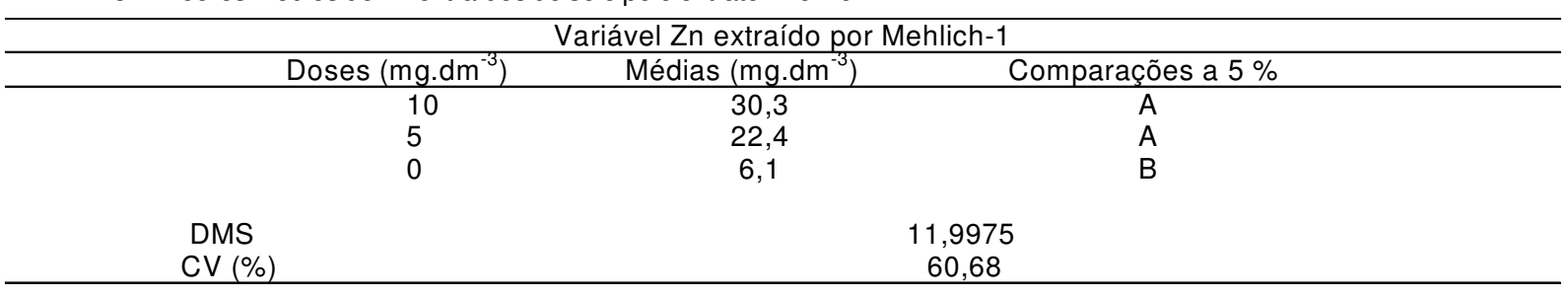

Letras diferentes nas colunas diferem entre si pelo teste de Tukey a $5 \%$ de probabilidade.

Os valores de Zn extraídos por DTPA (Tabela 4) demonstraram diferenças significativas também entre a testemunha e os tratamentos 5 e $10 \mathrm{mg} \mathrm{dm}^{-3}$. Pode- se verificar que os teores de Zn disponível no solo, tanto para Mehlich-1 como para DTPA, apresentaramse de forma crescente conforme o incremento da dose.

TABELA 4 - Teores médios de Zn extraídos do solo pelo extrator DTPA.

\begin{tabular}{cccc}
\hline \multicolumn{3}{c}{ Variável Zn extraído por DTPA } \\
\hline & Doses $\left(\mathrm{mg}^{-3} \mathrm{dm}^{-3}\right)$ & Médias $\left(\mathrm{mg} \cdot \mathrm{dm}^{-3}\right)$ & Comparações a 5\% \\
\hline & 10 & 26,8 & $\mathrm{~A}$ \\
& 5 & 17,9 & $\mathrm{~B}$ \\
DMS & 0 & 2,8 & \\
CV $(\%)$ & & 10,8567 & \\
& & & 67,72 \\
\hline
\end{tabular}

Letras diferentes nas colunas diferem entre si pelo teste de Tukey a $5 \%$ de probabilidade.

Nas três doses de $\mathrm{Zn}$ pode se verificar que o Mehlich se sobressaiu quando comparado ao DTPA (Tabelas 3 e 4). Segundo Galrão (5), pode ocorrer influência do próprio extrator no $\mathrm{pH}$ do solo. Assim extratores ácidos como o Mehlich-1, podem extrair mais $\mathrm{Zn}$ do solo, podendo extrair níveis superiores ao disponível para a planta, já os extratores complexantes como o DTPA, extraem menos Zn do solo, apontando para maior eficácia do primeiro em solos mais ácidos e do segundo em solos alcalinos.

A concentração do $\mathrm{Zn}$ disponível pode ser explicada devido ao $\mathrm{pH}$ ácido determinado no solo (Tabela 1), pois segundo Brady (3), a grande variabilidade dos solos é um fator importante na diferenciação da concentração de Zn disponível de um solo para outro, sendo a disponibilidade e a solubilidade altamente dependentes do $\mathrm{pH}$, ao ponto que para cada unidade de aumento deste, a concentração de $\mathrm{Zn}$ na solução de solo decresce 100 vezes.

A análise de variância demonstrou diferença estatística nos teores de $\mathrm{Zn}$ em função das doses aplicadas com relação à fitodisponibilidade (Tabela 5). Com o incremento das doses de $\mathrm{Zn}$ no solo, independentemente da adubação NPK, houve acréscimos significativos de $\mathrm{Zn}$ no tecido foliar. Korndörfer (9) também verificou que os teores de $\mathrm{Zn}$ na folha do milho aumentaram significativamente com as doses de $\mathrm{Zn}$ aplicada. No tratamento com $10 \mathrm{mg}$ $\mathrm{dm}^{-3}$, ocorreu a maior disponibilidade do elemento para a planta.

TABELA 5 - Teores médios de Zn no tecido foliar (fitodisponibilidade) em função das doses deste micronutriente.

\begin{tabular}{ccc}
\hline Doses de $\mathrm{Zn}\left(\mathrm{mg} \cdot \mathrm{dm}^{-3}\right)$ & Comparações a $5 \%$ \\
\hline 10 & 35,87 & $\mathrm{~A}$ \\
5 & 29,10 & $\mathrm{~B}$ \\
0 & 23,93 & $\mathrm{C}$
\end{tabular}

$$
\text { CV (\%) }
$$

$14,285 \%$

Letras diferentes nas colunas diferem entre si pelo teste de Tukey a $5 \%$ de probabilidade.

De acordo com Buzetti (4), o teor de Zn nas plantas varia bastante, dependendo das espécies e de fatores do solo. Normalmente, os teores encontramse na faixa de 20 a $50 \mathrm{mg} \cdot \mathrm{dm}^{-3}$, sendo freqüentes sintomas de deficiência quando as plantas apresentam teores menores do que $20 \mathrm{mg} \cdot \mathrm{dm}^{-3}$.

Assim, pode-se verificar na tabela 5 que os teores de $\mathrm{Zn}$ no tecido foliar encontram-se na faixa verificada normalmente nas plantas. $\mathrm{Na}$ dose $0 \mathrm{mg}^{-\mathrm{dm}^{-3}}$ de $\mathrm{Zn}$ verifica-se teores adequados do elemento no tecido foliar, demonstrando possivelmente que o teor original do solo já apresentava índices adequados.

Observando as figuras 1 e 2 nota-se que ambos os extratores proporcionam correlação entre $\mathrm{Zn}$ extraído do solo com o $\mathrm{Zn}$ acumulado na parte aérea da planta, com valor maior de $\mathrm{R}^{2}$ para o DTPA. 


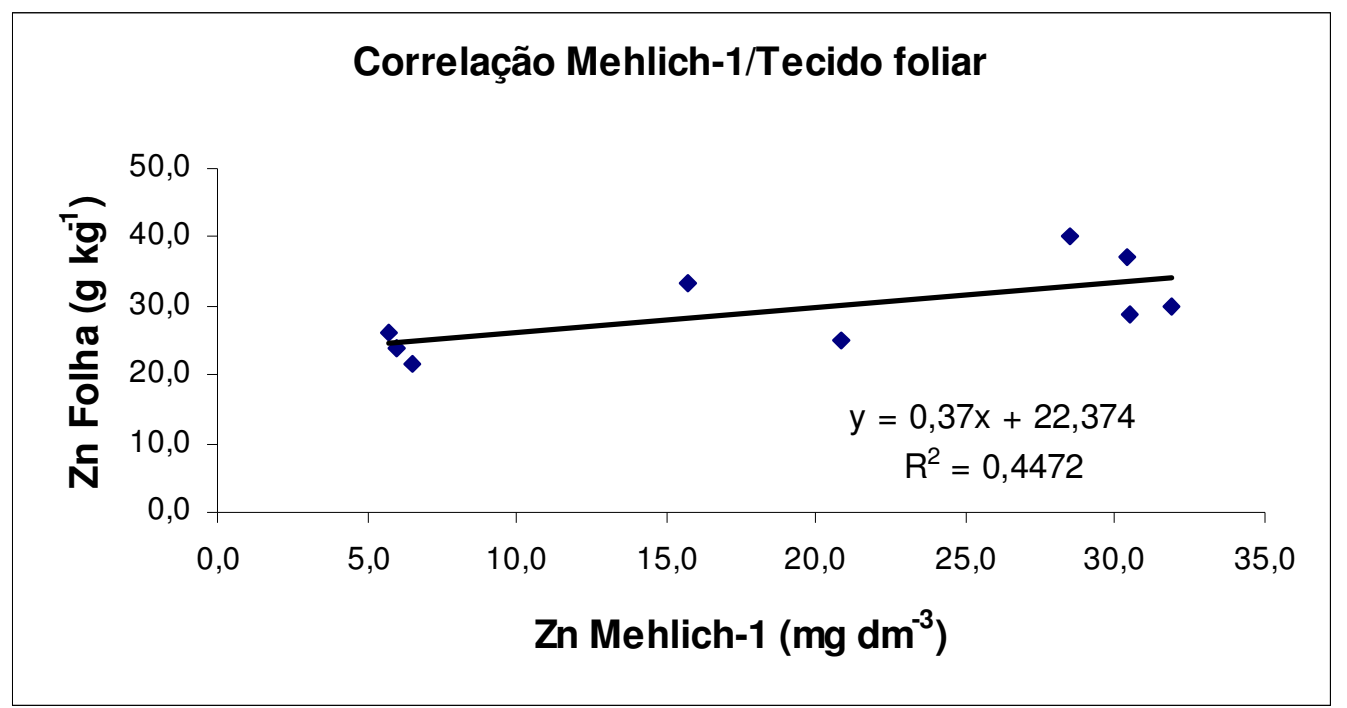

FIGURA 1 - Correlação entre os teores de Zn extraídos pelo método Mehlich-1e teores de Zn presentes no tecido foliar do milho.

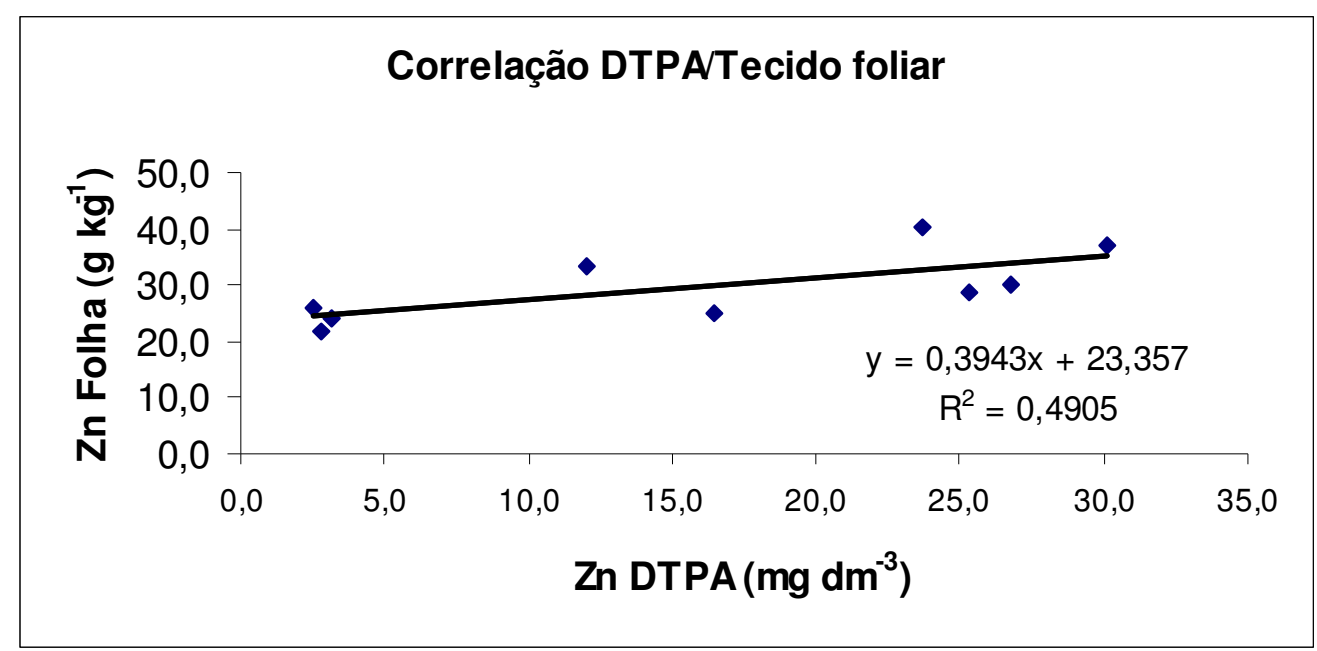

FIGURA 2 - Correlação entre os teores de Zn extraídos pelo método DTPA teores de Zn presentes no tecido foliar do milho.

De acordo com Abreu e Raij (1), que estudaram o efeito da reação do solo no Zn extraído pelas soluções de DTPA e Mehlich-1, o extrator DTPA mostrou melhores resultados com valores altos e positivos de correlação entre os teores de $\mathrm{Zn}$ na planta e no solo que o extrator Mehlich-1. Concluindo que a mudança na disponibilidade de $\mathrm{Zn}$, decorrente da reação do solo, foi mais bem avaliada pela solução de DTPA.

Os resultados apresentados para os extratores são médias compostas dos valores do elemento extraídos pelo Mehlich - 1 e DTPA, oriundos de amostras coletadas nas linhas e entrelinhas, com o objetivo de se obter uma média geral para parcela. Correlacionando-se os valores de $\mathrm{Zn}$ obtidos pelos extratores, observa-se uma alta relação entre ambos os métodos, sendo o $\mathrm{R}^{2}$ igual a $98 \%$ sugerindo pouca diferença entre os métodos de extração (Figura 3). Ao se analisar a figura 3 , nota-se que os valores extraídos pelo DTPA e Mehlich foram altamente significativos. Portanto, ambos os extratores podem ser utilizados na determinação dos teores de Zn em solos de textura média. 


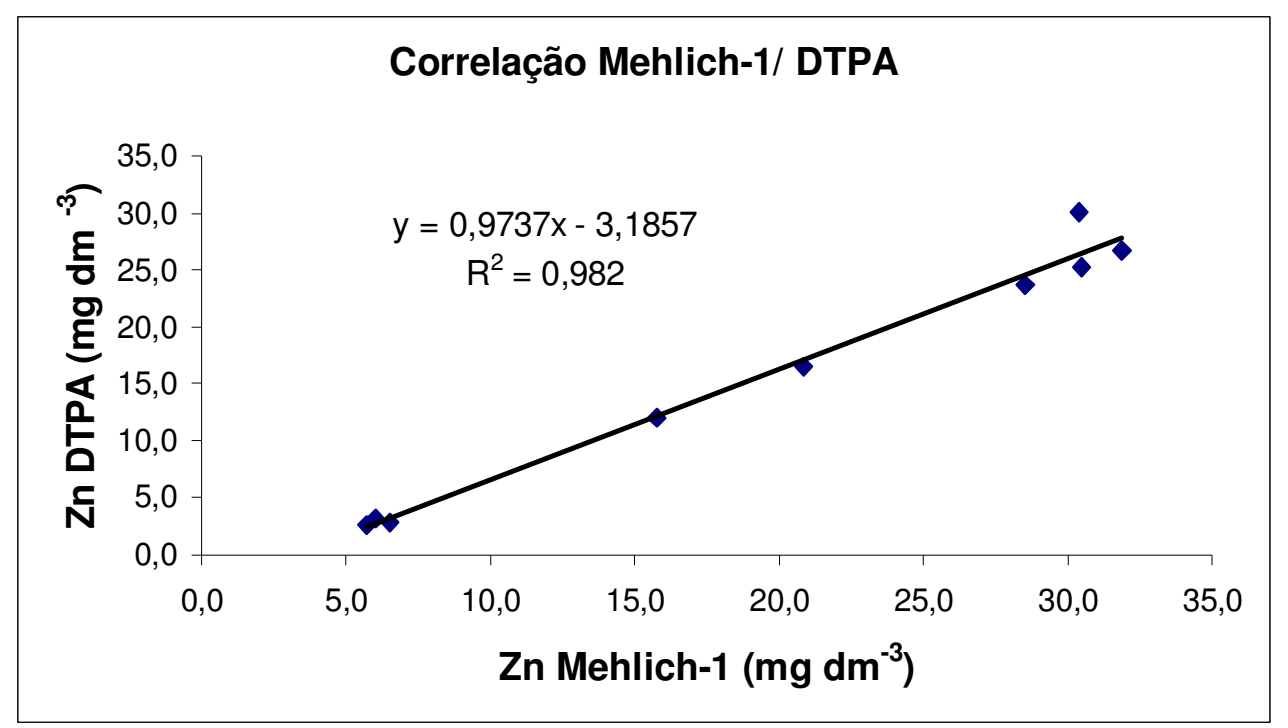

FIGURA 3 - Correlação entre os teores de Zn extraídos do solo por Mehlich-1e DTPA.

\section{CONCLUSÃO}

Os extratores Mehlich-1 e DTPA demonstraram uma boa correlação entre os teores de $\mathrm{Zn}$ presentes no solo e sua fitodisponibilidade no tecido foliar do milho. Os valores de $\mathrm{Zn}$ extraídos por Mehlich-1 e DTPA foram altamente significativos mostrando a excelente correlação entre os mesmos para este solo.

\section{REFERÊNCIAS}

1. ABREU, C.A. \& RAIJ, B.V. Efeito da reação do solo no zinco extraído pelas soluções de DTPA e Mehlich-1. Campinas: Bragantia, v. 55, p. 357-363, 1996

2. BATAGLIA, O.C. \& RAIJ, B.V. Eficiência de extratores de micronutrientes na análise de solo. Campinas: Revista Brasileira Ciência do Solo, v. 13, p. 205-212, 1989.

3. BRADY, N.C. Natureza e propriedade dos solos. Rio de Janeiro: Freitas Bastos, p. 506, 1983.

4. BUZETTI, S. Estudo da eficiência de extratores químicos de zinco, no solo, para milho. Campinas: Revista Brasileira Ciência do Solo, v. 16, p. 367-372. 1991.

5. GALRÃO, E.Z. Níveis críticos de zinco para o milho cultivado em Latossolo Vermelho-Amarelo, fase cerrado. Revista Brasileira Ciência do Solo, v. 19, p. 255-260, 1995.

6. HENDRICKSEN, R.E.; GILBERT, M.A. \& PUNTER, L.D. Effect of superphosphate application on macronutrient and micronutrient concentrations in grazed stylonative grass pasture in tropical Austrália. Aust. J. Agric. Rés, v. 43, p. 17251738, 1992.

7. IAPAR, Instituto Agronômico do Paraná. Análise química de tecido vegetal. Londrina: IAPAR, 1992. 17 p. Circular, 74.

8. IAPAR, Instituto Agronômico do Paraná. Manual de análise química de solo e controle de qualidade. Londrina: IAPAR, 1992. 40 p. Circular 76.

9. KORNDÖRFER, G.H.; ALCANTARA, C.B.; HOROWITZ, N. \& LANA, R.M.Q. Formas de adição de zinco a um formulado NPK e seu efeito sobre a produção de milho. Revista Scientia Agrícola, v. 52, n. 3. 1999.

10. LUCHESE, E.B.; LENZI, E.; RAUBER, T.; NAKASHIMA, P. \& GALLI, D. Levantamento preliminar dos teores de zinco e ferro nos solos do Paraná. Arq. Biol. Tcnol., Curitiba, v. 33, p. 691-700, 1990.

11. MALAVOLTA, E. Manual de química agrícola: adubos e adubação. 3 ed. São Paulo: Agronômica Ceres, 1981.

12. McLAUGHLIN, M.J.; ZARCINAS, B.A.; STEVENS, D.P. \& COOK, N. Soil Testing for heavy metais. Comm. Soil Sei. Plant . Anal., v. 31, p. 1661-1700, 2000.

13. OLIVEIRA, M.E.G.; NOVAIS, R.E; NEVES, J.C.L.; VASCONCELOS, C.A. \& ALVES, V.M.C. Relação entre o zinco "disponível”, por diferentes extratores, e as frações de zinco em amostras de solos. Revista Brasileira Ciência do Solo, v. 23, p. 827-836, 1999.

14. TOMÉ JR, J.B. Manual para Interpretação de Análise de Solo. Guaíba: Agropecuária Ltda. 1997. 240 p.

Recebido em 30/04/2004 Aceito em 25/07/2006 